\title{
Application of Liesegang Ring Formation on a Gelatin Film to the Determination of Sulfate Concentration in Individual Rain Droplets
}

\author{
Ken'ichi Narita, Kiyoshi Matsumoto, ${ }^{\dagger}$ and Manabu Igawa \\ Department of Applied Chemistry, Faculty of Engineering, Kanagawa University, \\ 3-27-1, Rokkakubashi, Kanagawa, Yokohama 221-8686, Japan
}

\begin{abstract}
The determination of $\mathrm{SO}_{4}{ }^{2-}$ concentration in individual rain droplets can be achieved using a gelatin film containing 5 $\mathrm{mM}$ barium chloride and $5 \mathrm{mM}$ barium nitrate. $\mathrm{SO}_{4}{ }^{2-}$ in droplets reacts with barium chloride in the film to form the characteristic Liesegang rings. The areal ratio of the ring to the droplet print (ARRD) on the film is a good indicator for determining the $\mathrm{SO}_{4}{ }^{2-}$ concentrations in an individual droplet. Interference by $\mathrm{NO}_{3}{ }^{-}$in determining $\mathrm{SO}_{4}{ }^{2-}$ concentration can be avoided by adding barium nitrate to the gelatin. Measurements of $\mathrm{SO}_{4}{ }^{2-}$ in rainwater samples by this method agreed well with those from ion chromatography. The individual analysis of rain droplets by this method indicated that the concentrations of $\mathrm{SO}_{4}{ }^{2-}$ were largely different with a 5-order magnitude, even among individual rain droplets collected simultaneously, and were higher in smaller rain droplets.
\end{abstract}

(Received June 14, 2006; Accepted September 7, 2006; Published December 10, 2006)

\section{Introduction}

Individual analysis of small droplets, such as rain and fog droplets, has been rarely reported, although the chemical components and their concentrations in droplets would be important to understand the physicochemistry of cloud, fog, and/or rain processes. Only a few researchers have achieved this by applying a fixation technique with the exposure of liquid nitrogen ${ }^{1}$ or cyanoacrylate vapor $^{2}$ to droplets before chemical analysis.

A reagent-coated thin-film method developed by Bigg et al. ${ }^{3}$ has been widely applied to the qualitative analysis of chemical components in tropospheric and stratospheric aerosols. A thin film of a reagent mounted on an electron microscope screen by vacuum evaporation has been used in this method. After collecting aerosols on a thin film, the reaction products were examined with an electron microscope to identify the chemical components in the aerosols. Individual particle analysis using the reagent-coated thin-film method has contributed to important findings in aerosol chemistry, such as the chemical composition of stratospheric aerosols ${ }^{4}$ and the internal mixing status of tropospheric aerosols. ${ }^{5}$ Thin films of barium chloride have been used to detect sulfate in aerosols that react with barium chloride, and then form characteristic reaction rings, called Liesegang rings. ${ }^{3}$ This is still a useful method for detecting sulfate in aerosols individually, but is hardly applied to quantitative analysis. Only a few pioneering researchers have reported experimental calibration curves between the diameters of the reaction ring and the masses of sulfate in individual particles with diameters of $0.1-2 \mu \mathrm{m} .{ }^{6,7}$

In order to develop the quantitative analysis of ionic species in

$\doteqdot$ To whom correspondence should be addressed.

E-mail: ft101666@kanagawa-u.ac.jp individual small droplets, such as rain and fog droplets, we applied a film of gelatin containing reagents to determine the $\mathrm{SO}_{4}{ }^{2-}$ concentration in a small droplet. In past studies, a gelatin film method has been reported to detect ionic species, such as sulfate and chloride in micron and submicron particles qualitatively. ${ }^{8,9}$ Although Farlow ${ }^{10}$ has reported a quantitative determination of the masses of chloride in sodium chloride particles by a gelatin film containing silver salt, the present paper reports on the first quantitative determination of the concentration of target ions in individual sample droplets by the gelatin film method.

\section{General Method}

A gelatin solution $(5 \% \mathrm{w} / \mathrm{w})$ containing $5 \mathrm{mM}$ barium chloride was dropped on a slide glass, spread thinly and flatly using a glass stick, and then rapidly dried by blowing hot air. Hereby, a flat film of gelatin containing barium chloride with a thickness of $1.0-3.5 \mu \mathrm{m}$ was mounted on the slide glass.

A droplet of sample solution including $\mathrm{SO}_{4}{ }^{2-}$ was dropped on the film and the slide glass was then stored in a desiccator for 4 days at a constant relative humidity of $80 \%$. In this study, $\mathrm{SO}_{4}{ }^{2-}$ in the sample solution was prepared with $\mathrm{Na}_{2} \mathrm{SO}_{4}$. After that, the ring was examined by a digital optical microscope (Sonic Co. Ltd., BS-D8000II). Barium chloride in the film reacted with $\mathrm{SO}_{4}{ }^{2-}$ in the sample droplet, and then formed Liesegang rings, of which the representative shape is shown in Fig. 1.

In our preliminary experiments, the barium chloride concentrations in the gelatin solution were changed under the conditions of $\mathrm{SO}_{4}{ }^{2-}$ concentration between 0.1 and $300 \mu \mathrm{M}$ in the sample droplets. These concentrations of $\mathrm{SO}_{4}{ }^{2-}$ are in a possible range for ambient rainwater. Barium chloride with 5 $\mathrm{mM}$ was the most suitable to form the rings clearly in all cases of $\mathrm{SO}_{4}{ }^{2-}$ concentrations from 0.1 to $300 \mu \mathrm{M}$. In the case of 


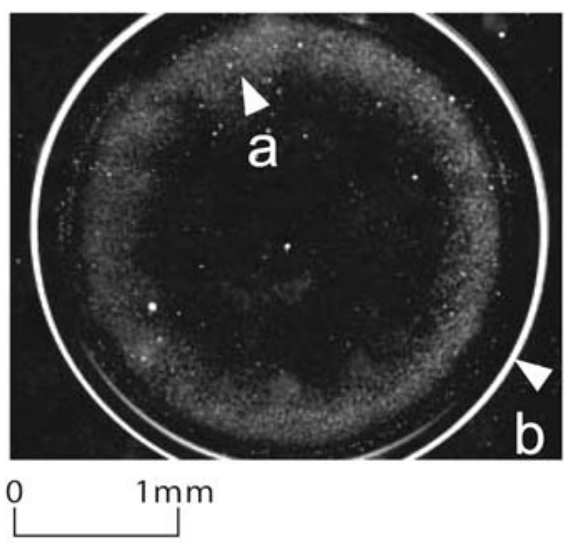

Fig. 1 Photograph of the representative Liesegang ring (a) and the edge of the droplet print (b).

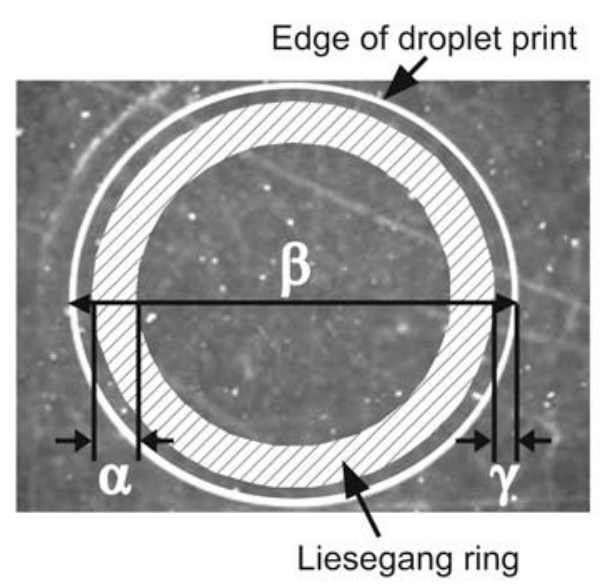

Fig. 2 Schematic diagram of the Liesegang ring. $(\alpha)$ Width of the ring, $(\beta)$ diameter of the droplet print, $(\gamma)$ distance between the edge of the droplet print and the outer edge of the ring.

higher concentrations of barium chloride, no ring was found under the conditions of low $\mathrm{SO}_{4}{ }^{2-}$ concentrations, probably because the extreme overload of barium chloride prevented the development of the ring. In the case of lower concentrations of barium chloride, on the other hand, the ring did not appear under the conditions of high $\mathrm{SO}_{4}{ }^{2-}$ concentrations, probably due to a lack of barium chloride.

The effects of the concentration of gelatin in the solution and the thickness of the film were also evaluated in preliminary experiments. In order to observe the ring clearly, the film with a gelatin concentration of $5 \%(\mathrm{w} / \mathrm{w})$ was the most suitable and its thickness should be controlled to $5 \mu \mathrm{m}$ or less. Moreover, the relative humidity was also changed from 40 to $100 \%$, showing that a relative humidity of $80 \%$ is the best condition for storage to form the ring clearly.

\section{Laboratory Test}

Availability of the areal ratio of the ring to the droplet print

The area of the ring is in proportion to the amount of $\mathrm{SO}_{4}^{2-}$ in the sample droplet, and the area of the droplet print, which is a mark of the sample droplet remaining on the film after drying (see Fig. 1), is in proportion to the volume of the sample droplet. Therefore, the areal ratio of the ring to the droplet print

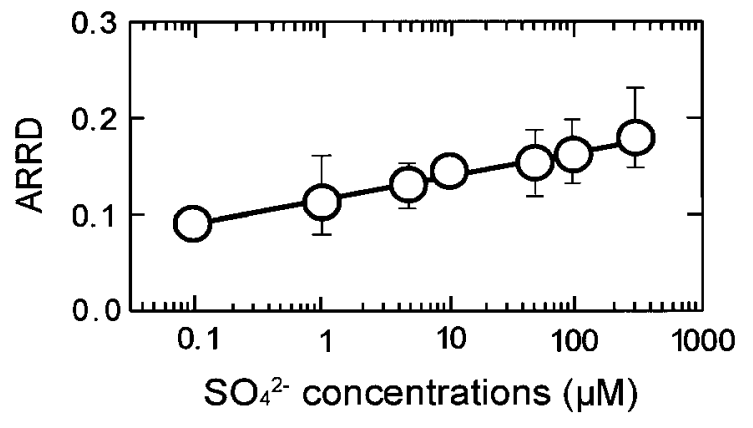

Fig. 3 Relationship between the $\mathrm{SO}_{4}{ }^{2-}$ concentration in the sample droplet and the areal ratio of the ring to the droplet print (ARRD) of the Liesegang rings formed on gelatin film containing $5 \mathrm{mM}$ barium chloride. The error bars indicate standard deviations.

(ARRD) is expected to be correlated with the $\mathrm{SO}_{4}{ }^{2-}$ concentrations in the sample droplet. The width of the ring $(\alpha)$, the diameter of the droplet print $(\beta)$, and the distance between the edge of the droplet print and the outer edge of the ring $(\gamma)$, of which a schematic diagram is shown in Fig. 2, were measured at several points on one ring, and then averaged to calculate the area of the ring and the droplet print. The area of the ring $(R)$ and the droplet print $(D)$ can be represented by the following expressions:

$$
\begin{aligned}
& R=\pi\left[\{(\beta / 2)-\gamma\}^{2}-\{(\beta / 2)-(\alpha+\gamma)\}^{2}\right], \\
& D=\pi(\beta / 2)^{2} .
\end{aligned}
$$

Therefore, the ARRD can be calculated as follows:

$$
\mathrm{ARRD}=R / D=\left[\{(\beta / 2)-\gamma\}^{2}-\{(\beta / 2)-(\alpha+\gamma)\}^{2}\right] /(\beta / 2)^{2} .
$$

The ARRD values were well correlated with the concentrations of $\mathrm{SO}_{4}{ }^{2-}$ in the sample droplet, as shown in Fig. 3 . This means that the ARRD would be useful for determining the concentrations of $\mathrm{SO}_{4}{ }^{2-}$ in the sample droplet, although the reason why the $\mathrm{SO}_{4}{ }^{2-}$ concentrations can be expressed as logarithmic values for the linear regression remains an unresolved question.

In order to make sure that the volume of the sample droplet does not affect the relationship, the volume was changed from 0.1 to $5 \mu \mathrm{l}$, which corresponds to the representative diameters of ambient rain droplets from 0.6 to $2.1 \mathrm{~mm}$, with the concentration of $\mathrm{SO}_{4}{ }^{2-}$ fixed at $50 \mu \mathrm{M}$. The ARRD was not changed with the volume, indicating no influence of the volume on the ARRD. Although the changes in the amount of $\mathrm{SO}_{4}{ }^{2-}$ and the volume of the sample droplet are related to the changes in the area of the ring and the droplet print, respectively, the ARRD values were correlated just with the concentrations of $\mathrm{SO}_{4}{ }^{2-}$.

\section{Interference by coexisting ions}

A determination of the $\mathrm{SO}_{4}{ }^{2-}$ concentration in ambient rainwater was attempted with a gelatin film using the ARRD. Ambient rainwater was collected by an automatic rainwater collector (Konan Kaihatsu, Ltd.). ${ }^{11}$ Table 1 shows the measurements of $\mathrm{SO}_{4}{ }^{2-}$ in rainwater obtained at the early (30 min after the rain started) and late stages (30 min before the rain stopped) of a rainfall event. The measurements of $\mathrm{SO}_{4}{ }^{2-}$ by ion chromatography, of which the conditions were reported in our previous paper, ${ }^{12}$ are also listed in the table to verify the 
Table 1 Measurements of $\mathrm{SO}_{4}{ }^{2-}$ in rainwater obtained at the early and late stages of a rainfall event on 5 December, 2002, by the gelatin film method ( $5 \mathrm{mM}$ barium chloride) and ion chromatography (unit; $\mu \mathrm{M}$ )

\begin{tabular}{lcc}
\hline Analytical method & Early stage & Late stage \\
\hline This method & $0.32 \pm 0.015$ & $5.0 \pm 0.012$ \\
Ion chromatography & 48.0 & 6.0 \\
\hline
\end{tabular}

accuracy of the gelatin film method. Although a good agreement between both methods was found in the sample from the late stage of a rainfall event, an extremely lower concentration was obtained by the gelatin film method in the sample from the early stage of a rainfall event. Because various ionic species would exist at higher concentrations in rainwater from the early stage of a rainfall event, it is expected that some coexisting ions prevent the development of a ring due to their high concentrations.

In order to evaluate the influence of coexisting ions on the quantitative measurement of $\mathrm{SO}_{4}^{2-}$, sample solutions of $\mathrm{SO}_{4}^{2-}$ including other ionic species $\left(\mathrm{NH}_{4}^{+}, \mathrm{Mg}^{2+}, \mathrm{Ca}^{2+}, \mathrm{Cl}^{-}, \mathrm{NO}_{3}^{-}\right.$, $\mathrm{CO}_{3}{ }^{2-}$, and $\mathrm{CH}_{3} \mathrm{COO}^{-}$) were dropped on the film. These ions are usually contained in ambient rainwater at relatively high concentrations. Figure 4 shows the results for the cases of $\mathrm{NH}_{4}^{+}, \mathrm{Cl}^{-}$, and $\mathrm{NO}_{3}{ }^{-}$. Here, the $\mathrm{SO}_{4}{ }^{2-}$ concentration in the sample solutions was fixed at $50 \mu \mathrm{M}$, whereas other ion concentrations were changed from 1 to 50 or $100 \mu \mathrm{M}$. The ARRD decreased with the $\mathrm{NO}_{3}{ }^{-}$concentration, but no influence on the ARRD was found in the cases of $\mathrm{NH}_{4}{ }^{+}$and $\mathrm{Cl}^{-}$. Other ionic species of $\mathrm{Mg}^{2+}, \mathrm{Ca}^{2+}, \mathrm{CO}_{3}{ }^{2-}$, and $\mathrm{CH}_{3} \mathrm{COO}^{-}$also did not show any influence on the ARRD. Coexisting $\mathrm{NO}_{3}{ }^{-}$in the sample solution could prevent the development of the ring of barium sulfate precipitation, probably due to the formation of barium nitrate in the film, which could lead to an underestimation of the $\mathrm{SO}_{4}{ }^{2-}$ concentration in the rainwater from the early stage of a rainfall event.

\section{Quantitative analysis of $\mathrm{SO}_{4}{ }^{2-}$}

To avoid the influence by $\mathrm{NO}_{3}{ }^{-}$on the formation of the ring, barium nitrate was added to the gelatin. The gelatin film containing $5 \mathrm{mM}$ barium chloride and $5 \mathrm{mM}$ barium nitrate was prepared and first applied to a sample solution including only $\mathrm{SO}_{4}{ }^{2-}$. The ARRD values were well correlated with the concentrations of $\mathrm{SO}_{4}^{2-}$ from 0.1 to $1000 \mu \mathrm{M}$ in the sample droplet, and were not affected by the volume of the solution as well as the case of the film containing only $5 \mathrm{mM}$ barium chloride.

Gelatin film containing $5 \mathrm{mM}$ barium chloride and $5 \mathrm{mM}$ barium nitrate was then applied to measure $\mathrm{SO}_{4}{ }^{2-}$ in the sample solution that also included $\mathrm{NO}_{3}{ }^{-}$. Figure 5 shows the relationship between the ARRD and $\mathrm{NO}_{3}{ }^{-}$concentrations in the solution with $\mathrm{SO}_{4}{ }^{2-}$ fixed at $50 \mu \mathrm{M}$. Interference by $\mathrm{NO}_{3}{ }^{-}$with the ARRD was not found, meaning that the film containing barium nitrate would be useful for determining the $\mathrm{SO}_{4}{ }^{2-}$ concentration in a sample solution including $\mathrm{NO}_{3}^{-}$at high concentrations.

Figure 6 shows the relationship between the ARRD and $\mathrm{SO}_{4}{ }^{2-}$ concentrations in a solution with $\mathrm{NO}_{3}{ }^{-}$. Here, the concentrations of $\mathrm{NO}_{3}{ }^{-}$were changed randomly $(10 \mu \mathrm{M}$ in the case of $1 \mu \mathrm{M}$ $\mathrm{SO}_{4}^{2-}, 5 \mu \mathrm{M}$ for $5 \mu \mathrm{M} \mathrm{SO}{ }^{2-}, 1 \mu \mathrm{M}$ for $10 \mu \mathrm{M} \mathrm{SO}_{4}^{2-}, 1000 \mu \mathrm{M}$ for $50 \mu \mathrm{M} \mathrm{SO}_{4}{ }^{2-}, 10 \mu \mathrm{M}$ for $100 \mu \mathrm{M} \mathrm{SO}_{4}{ }^{2-}$, and $50 \mu \mathrm{M}$ for 1000 $\mu \mathrm{M} \mathrm{SO}{ }^{2-}$ ), because the ratios of $\mathrm{SO}_{4}{ }^{2-} / \mathrm{NO}_{3}{ }^{-}$in rainwater are largely changed in the ambient atmosphere. The ratios of $\mathrm{NO}_{3}$ to $\mathrm{SO}_{4}{ }^{2-}$ in the solutions are also plotted in Fig. 6. A good
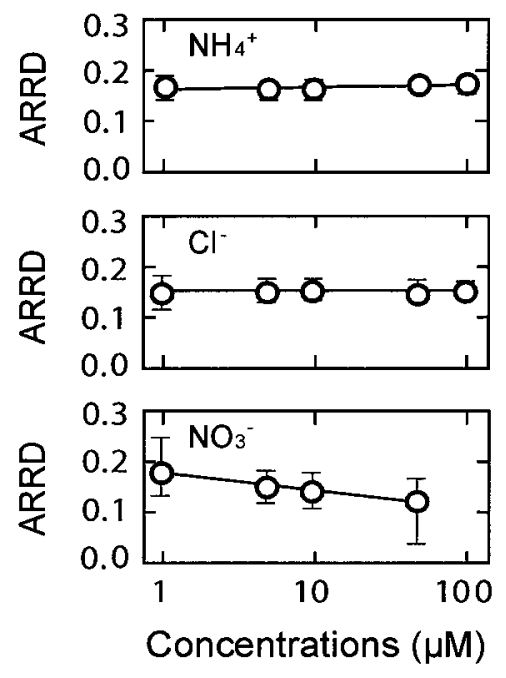

Fig. 4 Relationship between the concentrations of $\mathrm{NH}_{4}^{+}, \mathrm{Cl}^{-}$, and $\mathrm{NO}_{3}{ }^{-}$in the sample droplet and the ARRD of the Liesegang rings formed on gelatin film containing $5 \mathrm{mM}$ barium chloride. The concentration of $\mathrm{SO}_{4}{ }^{2-}$ was fixed at $50 \mu \mathrm{M}$. The error bars indicate standard deviations.

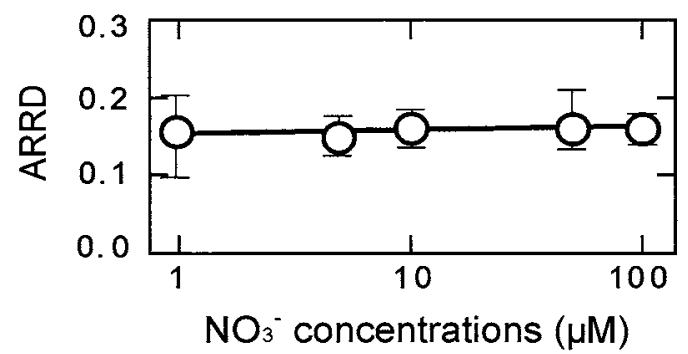

Fig. 5 Relationship between the $\mathrm{NO}_{3}{ }^{-}$concentration in the sample droplet and the ARRD of the Liesegang rings formed on gelatin film containing $5 \mathrm{mM}$ barium chloride and $5 \mathrm{mM}$ barium nitrate. The concentration of $\mathrm{SO}_{4}{ }^{2-}$ was fixed at $50 \mu \mathrm{M}$. The error bars indicate standard deviations.

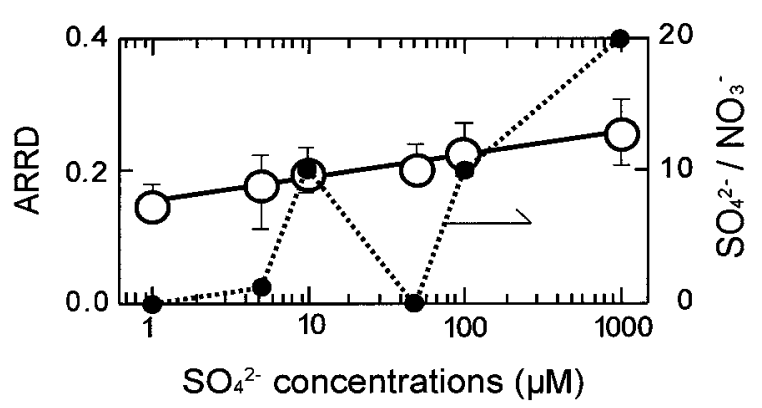

Fig. 6 Relationship between the $\mathrm{SO}_{4}{ }^{2-}$ concentration in the sample droplet and the ARRD of the Liesegang rings formed on gelatin film containing $5 \mathrm{mM}$ barium chloride and $5 \mathrm{mM}$ barium nitrate (open circles), and the ratios of the $\mathrm{SO}_{4}{ }^{2-}$ to $\mathrm{NO}_{3}{ }^{-}$concentration in sample droplets (closed circles). The error bars indicate standard deviations.

correlation with a straight regression line is found between the ARRD and the logarithmic values of the $\mathrm{SO}_{4}{ }^{2-}$ concentration, meaning that determination of the $\mathrm{SO}_{4}{ }^{2-}$ concentrations can be achieved without interference by $\mathrm{NO}_{3}{ }^{-}$, independently of the ratio of $\mathrm{SO}_{4}{ }^{2-} / \mathrm{NO}_{3}{ }^{-}$in the droplet. 
Table 2 Measurements of $\mathrm{SO}_{4}{ }^{2-}$ in rainwater obtained at the early and late stages of rainfall events on 6 and 27 March and 20 April, 2004 , by the gelatin film method ( $5 \mathrm{mM}$ barium chloride plus $5 \mathrm{mM}$ barium nitrate) and ion chromatography (unit; $\mu \mathrm{M}$ )

\begin{tabular}{|c|c|c|c|c|c|c|}
\hline \multirow{2}{*}{ Analytical method } & \multicolumn{2}{|c|}{6 Mar., 04} & \multicolumn{2}{|c|}{27 Mar., 04} & \multicolumn{2}{|c|}{20 Apr., 04} \\
\hline & Early stage & Late stage & Early stage & Late stage & Early stage & Late stage \\
\hline This method & $34.3 \pm 1.0$ & $9.1 \pm 1.1$ & $28.6 \pm 1.1$ & $18.2 \pm 1.0$ & $18.7 \pm 1.0$ & $5.1 \pm 1.1$ \\
\hline Ion chromatography & 35.5 & 8.5 & 27.1 & 18.7 & 18.7 & 9.6 \\
\hline
\end{tabular}

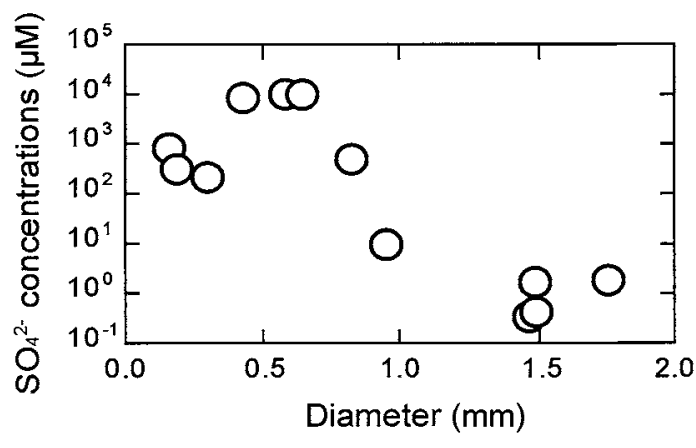

Fig. 7 Relationship between the $\mathrm{SO}_{4}{ }^{2-}$ concentration and the diameter of the ambient rain droplets.

Gelatin film containing $5 \mathrm{mM}$ barium chloride and $5 \mathrm{mM}$ barium nitrate was applied to determine $\mathrm{SO}_{4}{ }^{2-}$ in ambient rainwater. The measurements of $\mathrm{SO}_{4}^{2-}$ in rainwater obtained at the early and late stages of three rainfall events are summarized in Table 2. The measurements using the gelatin film method agreed well with those from ion chromatography in the cases of both the early and late stages of rainfall events. This suggests that the gelatin film containing $5 \mathrm{mM}$ barium chloride and 5 $\mathrm{mM}$ barium nitrate is useful for determining the $\mathrm{SO}_{4}{ }^{2-}$ concentration in droplets without any interference.

Our experiment showed that adding barium nitrate in a film of barium chloride could eliminate the interference by $\mathrm{NO}_{3}{ }^{-}$on the formation of a ring. The reason for this remained an unresolved question, although it is expected that the preexistence of $\mathrm{NO}_{3}{ }^{-}$in the film could eliminate the interference by reducing the effect of $\mathrm{NO}_{3}^{-}$in the droplets upon an increase of the $\mathrm{NO}_{3}{ }^{-}$ concentrations in the film.

\section{Field Application}

Gelatin film containing $5 \mathrm{mM}$ barium chloride and $5 \mathrm{mM}$ barium nitrate was applied to determine the $\mathrm{SO}_{4}{ }^{2-}$ concentrations in individual rain droplets. Some rain droplets in a rainfall event on 7 October, 2004, were directly captured on the film. The collection was conducted at the early stage of the rainfall event with a rainfall intensity of below $1 \mathrm{~mm} / \mathrm{h}$. The concentrations of $\mathrm{SO}_{4}{ }^{2-}$ in individual rain droplets are plotted versus the diameter of the droplets in Fig. 7. Because the area of the droplet print was correlated with the volume of the droplet, it could be converted to the diameter of the droplet.

This result shows that the concentrations of $\mathrm{SO}_{4}{ }^{2-}$ were largely different by 5 orders of magnitude, even among individual rain droplets collected simultaneously, although higher concentrations found in several droplets were out of the range with the certified linearity between the ARRD and $\mathrm{SO}_{4}{ }^{2-}$ concentrations and were therefore calculated by extrapolation of the regression line. Higher concentrations of $\mathrm{SO}_{4}{ }^{2-}$ were found in rain droplets with smaller diameters.

Rain droplets are formed through the processes of water vapor condensation on hygroscopic aerosols, subsequent growth of them by water vapor condensation, and collisions among small droplets with different diameters. These condensation and collision growth processes dilute the chemical species derived from the aerosols incorporated into rain droplets as cloud condensation nuclei. In addition, the adsorption of gaseous species into rain droplets and collisions of rain droplets with aerosols during their gravitational settling also contribute to the chemical species in rain droplets.

Higher concentrations of $\mathrm{SO}_{4}{ }^{2-}$ in smaller rain droplets mean that the adsorption processes of $\mathrm{SO}_{2}$ gas are not a dominant factor controlling the $\mathrm{SO}_{4}{ }^{2-}$ concentrations in rain droplets in this rainfall event. One reason for this can be attributable to the relatively low water solubility of $\mathrm{SO}_{2}$. It is expected that other species derived from more water-soluble gaseous species, for instance $\mathrm{NO}_{3}{ }^{-}$, could show somewhat different behavior from the case of $\mathrm{SO}_{4}{ }^{2-}$. In addition, it is also expected that the rain droplet chemistry in this rainfall event could be largely affected by a condensation growth process, which leads to an inverse correlation between the concentrations of $\mathrm{SO}_{4}{ }^{2-}$ and the diameter of rain droplets due to a dilution effect.

\section{Summary}

The determination of the $\mathrm{SO}_{4}{ }^{2-}$ concentration in individual small droplets has been achieved with a gelatin film containing $5 \mathrm{mM}$ barium chloride and $5 \mathrm{mM}$ barium nitrate. The areal ratio of the ring to the droplet print (ARRD) for the Liesegang rings formed on the film by the reaction of $\mathrm{SO}_{4}{ }^{2-}$ with barium chloride is well correlated with the $\mathrm{SO}_{4}{ }^{2-}$ concentrations in the sample droplet, which enables the determination of $\mathrm{SO}_{4}{ }^{2-}$ concentrations. A linearity between the ARRD and the logarithmic values of the $\mathrm{SO}_{4}{ }^{2-}$ concentration has been found in the range from 0.1 to $1000 \mu \mathrm{M}$, and therefore, the $\mathrm{SO}_{4}{ }^{2-}$ concentrations within this range can be determined quantitatively. Interference by $\mathrm{NO}_{3}{ }^{-}$in the analysis of $\mathrm{SO}_{4}{ }^{2-}$ can be avoided by adding barium nitrate in the gelatin. Measurements of rainwater samples on the gelatin film method agreed well with those from ion chromatography. The gelatin film method can be successfully applied to the determination of $\mathrm{SO}_{4}{ }^{2-}$ concentrations in ambient rain droplets individually without any interference.

The gelatin film method would be a useful and brief method for determining the $\mathrm{SO}_{4}{ }^{2-}$ concentration in individual ambient droplets, including not only rain droplets, but also fog droplets and mists, although its precision has significant uncertainties, as shown by the large scatter in the measured ARRD values. To improve our method more quantitatively, some future studies remain; for instance, the nonuniformity of the thickness of the film, which would be a significant source of scatter in the measured ARRD values, should be suppressed. 


\section{References}

Tjandradewi, and C. Takenaka, J. Meteor. Soc. Jpn., 1992,

1. K. Bächmann, I. Haag, U. Sprenger, K.-H. Steeg, K. 70, 711

6. Y. Mamane and R. G. Pena, Atmos. Environ., 1978, 12, 69. Steigerwald, B. Bastian, and A. Röder, Fresenius J. Anal.

7. G. P. Ayers, Atmos. Environ., 1978, 12, 1613. Chem., 1991, 340, 548.

2. M. Kasahara, Atmos. Res., 2003, 65, 251.

8. B. K. Seely, Anal. Chem., 1952, 24, 576.

9. B. K. Seely, Anal. Chem., 1955, 27, 93.

10. N. H. Farlow, Anal. Chem., 1957, 29, 883.

3. E. K. Bigg, A. Ono, and J. A. Williams, Atmos. Environ., 1974, 8,1 .

4. E. K. Bigg, J. Atmos. Sci., 1975, 32, 910.

5. G. W. Qian, Y. Ishizaka, Y. Minami, Y. Kurahashi, B. I.

11. M. Igawa, N. Nakada, and H. Okochi, J. Jpn. Soc. Atmos. Environ., 1999, 34, 211.

12. M. Takeuchi, H. Okochi, and M. Igawa, Atmos. Environ., 2004, 38, 4701. 